\section{Neuroendocrine tumor presenting as metastatic carcinoid and later as insulinoma: a rare metamorphosis}

\author{
Rabia Abdul Rehman, 1 \\ Richard Dale Childress,1,2 \\ Solomon S. Solomon, 1,3 \\ Hooman Oktaei1,2 \\ 1Department of Medicine \\ (Endocrinology), University of Tennessee \\ Health Science Center; ${ }^{2}$ Department of \\ Medicine (Endocrinology) and Research \\ Service, Veterans Administration Medical \\ Center of Memphis; ${ }^{3}$ Department of \\ Pharmacology, University of Tennessee \\ Health Science Center, TN, USA
}

\section{Introduction}

Gastroenteropancreatic neuroendocrine tumors (GEP-NETs) constitute a group of neoplasms that originate from the neuroendocrine cells. These tumors have secretory characteristics, and may present with distinct clinical syndromes although many remain clinically silent. Carcinoid tumors arise mostly from NETs of mid gut. Clinically, carcinoid syndrome causes dry flushing, bronchospasm, and tachycardia. ${ }^{1}$ Insulinomas are extremely rare neuroendocrine tumors, located almost exclusively in the pancreas. While the majority of the insulinomas are benign, less than $10 \%$ can be malignant with metastasis. They can also occur as part of familial syndromes such as multiple endocrine neoplasia type 1 and 2 (MEN $1 \& 2) .2,3$ Many patients present with metastatic disease with no identifiable primary site.

\section{Case Report}

We describe a very rare case of a neuroendocrine tumor, which initially presented as metastatic carcinoid with lesions in the liver and bones. Diagnosis was made by an elevated blood serotonin level and 24 hour urine 5HIAA. Liver biopsy confirmed the presence of a well differentiated neuroendocrine tumor (NET) staining positive for Chromogranin and negative for insulin. Two years after the initial diagnosis, the patient started to experience neuroglycopenic symptoms. Insulin and C-peptide levels were markedly elevated along with severe and recurrent hypoglycemic episodes. Due to extremely high insulin secretion from a large tumor burden, the patient's clinical condition rapidly deteriorated in spite of maximal medical treatment. Shortly after the diagnosis of metastatic Insulinoma with unknown primary was made, the patient developed multiple organ system failure which limited invasive diagnostic techniques for localization. It appears that this patient's neuroendocrine tumor had undergone a partial or complete change in its humoral function from a carcinoid or indolent neuroendcorine tumor to an Insulinoma or that the tumor started to co-secrete insulin along with serotonin. Less likely, he may have developed a separate tumor in the pancreas which was unable to be visualized by conventional imaging. Excess secretion of both Serotonin and Insulin from a single tumor is extremely rare and very few cases have been reported in the literature where a gastroenteopancreatic NET was found to secrete both insulin and serotonin.
A 57 year old white male presented to clinic with complaints of abdominal pain. He had a normal metabolic panel except for an alkaline phosphatase of 256 (38-126 U/L), alanine aminotransferase (ALT) 74 (0-40 IU/ L) and aspartate aminotransferase (AST) 67(055IU/L). Abdominal ultrasound and computerized tomography (CT) scan confirmed extensive metastatic disease in the liver, with the largest lesion $(10 \mathrm{~cm})$ located posteriorly in the right lobe. In particular, CT scan of the pancreas, chest and pelvis was normal. Further work up was significant for an elevated serotonin level, $414 \mathrm{ng} / \mathrm{mL}(26-165 \mathrm{ng} / \mathrm{mL})$. Twenty four hour urine 5HIAA was elevated at 15.2 (0.0-14.9 $\mathrm{mg} / 24 \mathrm{hr})$. Octreotide scan was markedly positive, with multiple lesions in the liver, bones and mediastinum. Liver biopsy done to assess the histology of the liver lesions confirmed a low grade neuroendocrine tumor. The immunoprofile of the tumor (chromogranin positive; CK20 positive; CK7 negative) supported this interpretation (Figure 1A). A diagnosis of carcinoid tumor with metastasis seemed likely. The patient was treated with monthly Sandostatin and remained asymptomatic from his carcinoid. He denied diarrhea, flushing, wheezing, dyspnea or rash. The patient had a prior history of prostate cancer (status post-prostatectomy) and hypertension. Additionally, patient was obese with a weight of $105.2 \mathrm{~kg}$ and body mass index of 35.6.

Two years later, he presented to his endocrinologist with complaints of feeling sweaty and shaky every morning for the last few months. He particularly noticed these episodes when he went without eating for few hours. His blood sugars during these episodes were
Correspondence: Rabia Abdul Rehman, University of Tennessee Health Science Centre 920 Madison Avenue, Suite 300A, Memphis, TN, USA. Tel. +1.901.6742144.

E-mail: rrehman@uthsc.edu

Key words: neuroendocrine tumors, insulinoma, carcinoid.

Acknowledgements: the authors would like to acknowledge Dr. David Spencer at Trumbull laboratories for his assistance.

Conflict of interests: the authors report no conflict of interests.

Received for publication: 17 August 2012.

Revision received: 28 September 2012.

Accepted for publication: 8 October 2012.

This work is licensed under a Creative Commons Attribution NonCommercial 3.0 License (CC BYNC 3.0)

(C) Copyright R.A. Rehman et al., 2012

Licensee PAGEPress, Italy

Endocrinology Studies 2012; 2:e7

doi:10.4081/es.2012.e7

always low in the range of 37 to $50 \mathrm{mg} / \mathrm{dL}$ (65$99 \mathrm{mg} / \mathrm{dL}$ ). He ate at frequent intervals to relieve his symptoms with subsequent elevation of his blood sugars. A detailed work up was ordered to evaluate cause of his fasting hypoglycemia (Table 1). The initial impression was that hypoglycemia was either due to simultaneous production of another hypoglycem from the metastatic carcinoid tumor or due to a new Insulinoma. In order to determine if insulin production from the metastatic carcinoid was present at the time of initial diagnosis, original liver biopsy slides were stained for Insulin. Insulin stain was negative (Figure 1B).

Due to persistent hypoglycemia symptoms, he was admitted to hospital. One cycle of chemoembolization of the liver lesions was performed utilizing strepotozocin as well as oral therapy with Afinitor. A repeat octreotide scan showed that some liver lesions were shrinking but some others on the right side were growing. Magnetic resonance imaging (MRI) of the pancreas showed a normal contour of the pancreas with normal signal intensity and no focal masses. Endoscopic ultrasound (EUS) to visualize any insulinoma that may have been missed on MRI could not be performed due to rapid deterioration of the patient's clinical condition. While in the hospital, the patient's plasma glucose values were running 33 and $32 \mathrm{mg} / \mathrm{dL}(65-99 \mathrm{mg} / \mathrm{dL})$. He required continuous infusion of high concentration dextrose fluids (D70 infusion) to keep his blood sugar above $60 \mathrm{mg} / \mathrm{dL}$. Maximal medical therapy was instituted with oral Diazoxide, 
Octreotide, Intravenous (IV) hydrocortisone and IV phenytoin to correct his hypoglycemia. Subsequently, his blood sugars showed some improvement along with a decrease in requirements of glucose containing fluids. Patient developed multiorgan failure and died during the same hospitalization.

\section{Discussion}

This report describes a rare case of a patient with GEP-NET, which initially presented with a relatively silent clinical presentation of a metastatic carcinoid, followed later by a serious hyperinsulinaemic hypoglycemic syndrome, leading to his demise. The primary site of NET was unidentified in this patient, despite multiple imaging modalities. The patient's liver biopsy specimens, obtained earlier in the course of his disease, stained positive for chromogranin $\mathrm{A}$ and negative for insulin. This along with the absence of hypoglycemia initially supports the fact that the patient did not have insulin hyper-secretion initially from these known liver metastases of the of the original carcinoid tumor. Although GEP-NETs secrete over 40 different hormones/proteins, simultaneous secretion of serotonin and insulin is extremely rare. A literature search has revealed only three similar cases of NETs. One case reported a primary hepatic neuroendocrine tumor, in which the humoral manifestations of the tumor changed during the course of the disease from extrapituitary acromegaly and carcinoid syndrome into a hyperinsulinemic hypoglycemic syndrome. ${ }^{4}$ In another case, ileal carcinoid with liver metastasis was proven to secrete serotonin, insulin, calcitonin and gastrin. ${ }^{5}$ Yet, another, similar case was reported of a metastatic pancreatic endocrine tumor secreting both insulin and serotonin. ${ }^{6}$ Conventional imaging modalities in our patient were unable to localize any pancreatic tumor and confirmation with EUS was not possible. The incidence of NETs in Caucasians is 4.44 per 100,000 persons per year. Carcinoid syndrome occurs in $20 \%$ of well-differentiated NETs in midgut. ${ }^{1}$ The incidence of Insulinoma is estimated at 4 per 1 million person-years. ${ }^{3}$ Statistically, it would be highly unlikely for two such rare tumors to exist in the same patient.

MEN1 is autosomal dominant predisposing to tumors of the parathyroid glands, anterior pituitary, and pancreatic islet cells. ${ }^{7}$ It is recognized that pancreatic islet cell tumors in MEN1 often synthesize multiple hormones, which could be because of defects in hormonal processing or inefficient secretory mechanisms. Nonfunctioning pancreatic neuroendocrine tumors may also occur with MEN1. ${ }^{8}$ Primary hyperparathyroidism is usually the initial
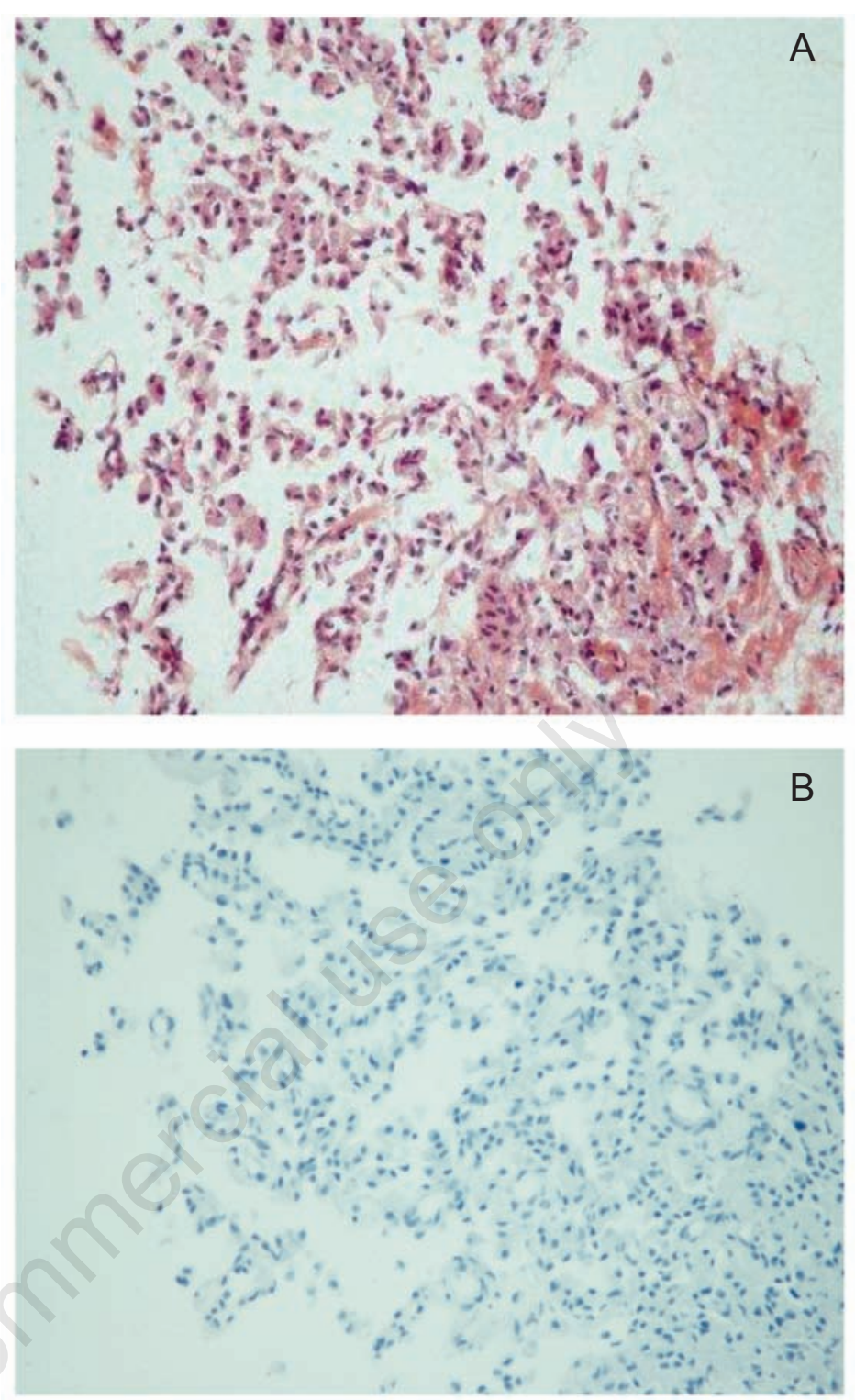

Figure 1. Haematoxylin/eosin-stained portion of patient's liver biopsy showing morphologically distinct population of cells with ample eosinophilic cytoplasm and round nuclei (A). These cells were positive for chromogranin and CK20 and negative for CK7 (not shown) and negative for Insulin immunohistochemical stain (B) (100× original magnification).

Table 1. Results of hormonal and biochemistry profile.

\begin{tabular}{lc}
\hline Endocrine tests & Concentration with reference ranges \\
Serum C-Peptide & $164(1.1-5.0 \mathrm{ng} / \mathrm{mL})$ \\
Insulin & $3938(0.0-29.1 \mathrm{uUmL})$ \\
\hline Proinsulin & $3897.2(0.0-9.4 \mathrm{pmol} / \mathrm{L})$ \\
Insulin antibodies & $2.2(\mathrm{uU} / \mathrm{mL})$ \\
\hline Beta hydroxyl butyric acid & $1.2(0.0-3.0 \mathrm{mg} / \mathrm{dL})$ \\
Sulfonylurea screen & Negative \\
\hline Growth hormone & $0.8(0-0.6 \mathrm{ng} / \mathrm{mL})$ \\
Insulin like growth factor II & $357(\mathrm{ng} / \mathrm{mL})$ \\
\hline Cortisol & $14.3(3.1-22.4 \mathrm{ug} / \mathrm{dL})$ \\
Chromogranin A & $2574.2(<36.4 \mathrm{ng} / \mathrm{mL})$ \\
\hline Glucose & $32(65-99 \mathrm{mg} / \mathrm{dL})$ \\
Creatinine & $4.0(0.5-1.4 \mathrm{mg} / \mathrm{dl})$ \\
\hline
\end{tabular}


manifestation of MEN1, which has 100\% penetrance after age 40 years. ${ }^{9}$ Although, our patient did not have a PTH level checked, his corrected total calcium level was in the range of 8.7-9.2 $\mathrm{mg} / \mathrm{dL}$, suggesting normal Parathyroid function and making MEN1 unlikely.

\section{Conclusions}

This case highlights the biochemical heterogeneity of the neuroendocrine tumors. Although rare, it is possible for a NET to change from an indolent tumor with a relatively silent clinical presentation to another much more aggressive one. Secondly, when NETs present with metastasis (even if non-functional), it is prudent to localize their primary site. This may be a challenge in cases where the primary tumor is very small and the patient very sick. Lastly, genetic mutations as in MEN1 should be kept in mind when dealing with atypical endocrine presentations.

\section{References}

1. Ramage JK, Ahmed A, Ardill J, et al. Guidelines for the management of gastroenteropancreatic neuroendocrine (including carcinoid) tumours (NETs). Gut 2012;61:6-32.

2. Cryer PE, Axelrod L, Grossman AB, et al. Evaluation and management of adult hypoglycemic disorders: an Endocrine Society Clinical Practice Guideline. J Clin Endocrinol Metabol 2009;94:709-28.

3. Service FJ, McMahon MM, O'Brien PC, Ballard DJ. Functioning insulinoma-incidence, recurrence, and long-term survival of patients: a 60-year study. Mayo Clinic Proc 1991;66:711-9.

4. Furrer J, Hattenschwiler A, Komminoth P, et al. Carcinoid syndrome, acromegaly, and hypoglycemia due to an insulin-secreting neuroendocrine tumor of the liver. J Clin Endocrinol Metabol 2001;86:2227-30.

5. Pelletier G, Cortot A, Launay JM, et al. Serotonin-secreting and insulin-secreting ileal carcinoid tumor and the use of in vitro culture of tumoral cells. Cancer 1984; 54:319-22.

6. Hinchliffe E, Allcock RL, Mansoor W, Myers MA. A patient with a metastatic gastroenteropancreatic endocrine carcinoma causing hyperinsulinaemic hypoglycaemia and the carcinoid syndrome. Ann Clin Biochem 2011;48:579-83.

7. Brandi ML, Gagel RF, Angeli A, et al. Guidelines for diagnosis and therapy of MEN type 1 and type 2. J Clin Endocrinol Metabol 2001;86:5658-71.

8. Newey PJ, Jeyabalan J, Walls GV, et al. Asymptomatic children with multiple endocrine neoplasia type 1 mutations may harbor nonfunctioning pancreatic neuroendocrine tumors. J Clin Endocrinol Metabol 2009;94:3640-6.

9. Marx SJ, Vinik AI, Santen RJ, et al. Multiple endocrine neoplasia type I: assessment of laboratory tests to screen for the gene in a large kindred. Medicine 1986;65:226-41. 